\title{
Editorial
}

\section{Awareness during anaesthesia: when is an anaesthetic not an anaesthetic?}

Alan R. Aitkenhead MD FRCA
Jones and Konieczko ${ }^{1}$ categorised the effects of progressively increasing concentrations of anaesthetic drugs on the brain crudely in four stages.

Stage 1. Conscious awareness without amnesia.

Stage 2. Conscious awareness with amnesia.

Stage 3. Subconscious awareness with amnesia.

Stage 4. No awareness.

Stage 1 of this series represents the state which patients and most doctors associate with the term "awareness during anaesthesia." It is more correctly termed "spontaneous recall" and is recognised by the ability of the patient to remember, with or without prompting, events which occurred during the period of anaesthesia. Stages 2 and 3 represent states in which the brain is able to respond either immediately (Stage 2) or at a later time (Stage 3) to verbal instructions or suggestions, but in which there is no subsequent spontaneous recall. The phenomenon of unconscious perception (Stage 3) has been demonstrated in a number of ways, although there have been contradictory findings and its clinical significance remains uncertain. ${ }^{2}$ However, spontaneous recall is of considerable clinical importance. It is confined almost entirely to patients who receive muscle relaxants together with inadequate amounts of anaesthetic drugs.

Reported incidences of spontaneous recall of intraoperative events vary widely. The most recent prospective study in which a structured interview was employed ${ }^{3}$ indicates that the incidence in non-obstetric and non-cardiac surgical practice in the United Kingdom is $0.2 \%$. In obstetric practice, the incidence is a little higher; ${ }^{4}$ this difference is attributable in part to a reluctance to use inhalational agents in adequate concentrations because of fears of inducing depression of the fetus and of increasing haemorrhage from the uterus,

From the University of Nottingham, U.K. and is probably due also to the short interval between induction of anaesthesia and skin incision in Caesarean section.

Much higher incidences of spontaneous recall have been reported in patients undergoing cardiac surgery, ranging from $1.14 \%^{3}$ to $23 \% .^{6}$ In a recent study ${ }^{7}$ investigating techniques which might be applicable for "fasttrack" cardiac surgical patients, the incidence of awareness was 3.3\%. In the present issue, a case of awareness during cardiac surgery is reported; ${ }^{8}$ the patient had recall of events during closure of the sternum, at a time when the end-tidal concentration of isoflurane was very low, when the brain concentration was probably still increasing after discontinuation of cardiopulmonary bypass and the re-institution of ventilation of the lungs with isoflurane, and when reliance was placed on a combination of sufentanil and midazolam to prevent awareness.

The principal reasons for the high incidence of spontaneous recall in patients undergoing cardiac surgery are that there is a tendency to rely heavily on opioids as a major component of the anaesthetic "cocktail," that conventional anaesthetic agents are avoided or administered in very low concentrations to avoid hypotension and that the usual sympathetic response to inadequate anaesthesia is often ablated by concurrent medication or by disease, making assessment of depth of anaesthesia more difficult. Recent studies involving the use of auditory evoked potentials and techniques designed to elicit conscious awareness with amnesia or unconscious perception may help to explain why spontaneous recall is more likely to occur when reliance is placed on the use of non-anaesthetic drugs to produced "anaesthesia." In addition, there is evidence that, in sub-MAC concentrations, anaesthetic drugs do not abolish spontaneous recall in equivalent proportions of their MAC values, indicating that not all anaesthetic drugs are equal in terms of their ability to abolish memory.

It is known that opioids are not anaesthetic agents. A 
ceiling effect occurs with fentanyl, ${ }^{9}$ sufentanil ${ }^{10}$ and alfentanil $^{11}$ in terms of their ability to reduce the MAC of inhaled agents or to produce adequate anaesthesia. During cardiac anaesthesia in which sufentanil was used alone for induction and maintenance (after premedication with morphine, scopolamine and diazepam), the P3a wave of the auditory event-related potential was present at various stages of the procedure, although the N1 wave was absent; ${ }^{12}$ this finding indicates that sufentanil decreases arousal but that discrimination of pitch change was present, probably as a result of the patients noticing the stimulus.

In order to maintain cardiovascular stability in cardiac surgery, many anaesthetists have turned to benzodiazepines rather than conventional anaesthetic agents to supplement opioids. However, while benzodiazepines often have an amnesic action, there is increasing evidence that they are not satisfactory anaesthetic agents. In a study of patients undergoing cardiac surgery, fentanyl was supplemented with nitrous oxide and flunitrazepam, isoflurane or propofol during maintenace of anaesthesia. ${ }^{13}$ Mid-latency auditory evoked potentials (MLAEPs) were reduced to a greater extent by propofol or isoflurane than by flunitrazepam. In another study, ${ }^{14}$ anaesthesia was maintained using high-dose fentanyl supplemented by flunitrazepam, isoflurane or propofol. MLAEPs were monitored, and an audiotape containing an implicit memory task was played. Seven of 30 patients demonstrated implicit recall. In all of these patients, but in only two of the remaining 23 , the latency of wave $\mathrm{Pa}$ increased by more than $12 \mathrm{~ms}$ and in all patients without recall, there was severe attenuation or abolition of the MLAEP waveform. Implicit recall occurred in 5/10 patients who received flunitrazepam, but only $2 / 20$ who received isoflurane or propofol.

Studies have been conducted also in patients undergoing non-cardiac procedures. During gynaecological surgery for which anaesthesia was induced with midazolam, diazepam or flunitrazepam, ${ }^{15}$ there were no marked changes in amplitude or latency of MLAEPs, indicating that primary cortical processing of auditory stimuli was preserved. In patients undergoing laparotomy, ${ }^{16}$ epidural analgesia was used to block painful sensation, and general anaesthesia was maintained with propofol, isoflurane or flunitrazepam and fentanyl. MLAEPs were severely attenuated or abolished in the first two groups, but not in the last. Purposeful movements or coughing, which were considered to be motor signs of wakefulness, occurred (significantly) more often in the patients who received flunitrazepam/fentanyl. Using the isolated forearm technique (a test of conscious awareness with amnesia), Russell ${ }^{17,18}$ found that $72 \%$ of patients responded to command during surgery when anaes- thetised with midazolam and alfentanil, $44 \%$ responded when receiving nitrous oxide and fentanyl, but only $7 \%$ responded when a continuous infusion of etomidate and fentanyl was used.

If it is accepted that the four stages described by Jones and Konieczko ${ }^{1}$ represent a continuum, then the inevitable conclusion must be that drug combinations which are associated with high incidences of conscious awareness or implicit recall, or with MLAEP waveform patterns found in patients with implicit recall, will be associated with an increased risk of spontaneous recall in a proportion of patients. Only volatile and intravenous anaesthetic agents suppress these responses.

To complicate matters further, the quantity of anaesthetic required to prevent spontaneous recall varies with the analgesic regimen employed, and may not be the same proportion of MAC (or, for intravenous anaesthetics, of the $\mathrm{EC}_{50}$ for suppression of movement) for all drugs. In unstimulated volunteers, Dwyer et al. ${ }^{19}$ showed that the $\mathrm{EC}_{50}$ to suppress memory was 0.20 MAC for isoflurane, but 0.50 MAC for nitrous oxide; end-tidal concentrations of $0.38 \mathrm{MAC}$ and $0.64 \mathrm{MAC}$ respectively prevented voluntary movement in response to command in $50 \%$ of volunteers. Nitrous oxide $70 \%$ reduces the plasma concentration of alfentanil required to suppress haemodynamic responses by about $80 \% .20,21$ However, nitrous oxide $67 \%$ reduces the plasma concentration of propofol required to prevent movement in response to surgical stimulation by only $33 \%$, yet reduces the MAC of volatile anaesthetic agents by a proportion which would be expected from the sum of the proportions of MAC administered. It may be better to consider nitrous oxide as primarily an analgesic drug rather than primarily an anaesthetic.

Does spontaneous recall matter? The answer is undoubtedly yes. Estimates of the proportion of patients who experience pain or discomfort during an episode of conscious awareness range from $10 \%{ }^{23}$ to $82 \% .^{24}$ Anybody who doubts the intensity of pain which can be experienced during surgery performed in the presence of inadequate anaesthesia should read the horrifying accounts which have been published. ${ }^{24,25}$ Hearing is the commonest sensation to be recalled; this often causes no distress, but can do so if the operating staff are heard to make uncomplimentary remarks about the patient, or if the conversation during the operation is apparently frivolous. Many patients are aware of being paralysed, and this frequently results in feelings of anxiety, panic, powerlessness and helplessness. ${ }^{26}$ The unexpected nature of these distressing experiences can result in the development of a traumatic neurosis, now referred to most commonly as the post-traumatic stress disorder. These patients develop anxiety attacks, flashbacks, nightmares 
and sleep disturbances, and may acquire a morbid fear of doctors and hospitals. In one study, ${ }^{26} 70 \%$ of patients with spontaneous recall developed psychological disturbances. It should be noted that in Blacher's original description of the traumatic neurosis associated with awareness, ${ }^{27}$ none of his patients had spontaneous recall; this reinforces my belief that we should not rely on amnesic agents such as the benzodiazepines to produce adequate anaesthesia.

Only about one-third of patients report an episode of spontaneous recall to their anaesthetist. Even when the episode is reported, medical staff are frequently unsympathetic and fail to manage the patient properly. ${ }^{28}$

The large majority of instances of awareness during anaesthesia result from an error in technique or in administration. ${ }^{29}$ However, at the present time, the precise concentration of anaesthetic agent required to guarantee lack of spontaneous recall is unknown. Nitrous oxide $70 \%$ (about $0.65 \mathrm{MAC}$ ) is insufficient. ${ }^{30}$ Isoflurane $1 \mathrm{MAC}$ prevents implicit learning during surgery $^{31}$ and probably exceeds the concentration required to prevent spontaneous recall. However, in balanced anaesthetic techniques which employ combinations of analgesic drugs, regional anaesthetic blocks, benzodiazepines and anaesthetic agents, the possible permutations are numerous, and the risk of spontaneous recall with each can be assessed only if large numbers of patients are studied prospectively. What appears highly likely is that a combination of drugs which prevents movement during surgery will prevent spontaneous recall associated with distress. When techniques are employed in which the risk of awareness is unknown, it would be prudent to use muscle relaxants to provide relaxation rather than paralysis, and to monitor the patient for signs of movement. If this is not feasible, then reliance should be placed on a technique with known efficacy in preventing spontaneous recall of intra-operative events.

\section{La conscience sous anesthésie ou quand un anesthésiste cesse de l'être}

Jones et Konieczko ${ }^{1}$ ont divisé en quatre stages fondamentaux les effets de l'augmentation des concentration anesthésiques au cerveau.
Stage 1. Conscience sans amnésie

Stage 2. Conscience avec amnésie

Stage 3. La demi-conscience sans amnésie

Stage 4. l'inconscience

La stage 1 de cette classification représente l'état que les patients et la plupart des médecins associent à l'expression «conscience sous anesthésie ». Les mots « rappel spontané » sont plus justes et on définit cet état par la capacité du patient de rapporter, avec ou sans incitation, ses souvenirs d'événements survenus pendant l'anesthésie. Les stages 2 et 3 représente des états au cours desquels le cerveau est capable de répondre immédiatement (stage 2) ou plus tardivement (stage 3) aux instruction verbales ou aux suggestions sans qu'il s'en souvienne nécessairement. Le phénomène de la perception inconsciente (stage 3) a été démontré de plusieurs façons, malgré certaines contradictions et sa signification demeure douteuse en clinique. ${ }^{2}$ Cependant, le rappel spontané a une grande importance en clinique. Il se limite presque entièrement aux patients qui ont reçu des relaxants musculaires avec des quantités insuffisantes d'anesthésiques.

L'incidence de rappel spontané d'événements peropératoires varie énormément. L'étude prospective la plus récente au cours de laquelle des entrevues structurées ont été utilisées ${ }^{3}$ révèle qu'au Royaume-Uni son incidence à l'exclusion de l'obstétrique et de la chirurgie cardiaque est de $0,2 \%$. En obstétrique, elle est un peu plus élevée. ${ }^{4}$ Cette différence est due notre répugnance à administrer des agents inhalatoires à des concentration suffisantes de crainte de déprimer le foetus et d'augmenter le risque d'hémorragie utérine et, possiblement, à cause de la brièveté de intervalle entre l'induction et l'incision de la peau lors d'une césarienne.

En chirurgie cardiaque, l'incidence de rappel spontané varie de $1,14 \% 5$ à $23 \% .^{6}$ Dans une étude récente en chirurgie cardiaque ${ }^{7}$ applicable à des patients de chirurgie cardiaque opérés expéditivement, l'incidence s'établissait de 3,3\%. Ce mois-ci, on rapporte dans le Journal un état de conscience pendant une intervention cardiaque. ${ }^{8}$ La patiente s'est souvenue des événements qui coïncidaient avec la fermeture du sternum, alors que la concentration télé-expiratoire d'isoflurane était à son plus bas, que la concentration cérébrale continuait peutêtre à augmenter après l'arrêt de la circulation extracorporelle et la relance de la ventilation pulmonaire avec l'isoflurane, et qu'on avait recours à une combinaison de sufentanil et de midazolam pour maintenir l'inconscience.

Cette forte incidence de rappel spontanée en chirurgie cardiaque est due à l'habitude que nous avons de faire 
appel à des morphiniques comme composants principaux du « cocktail : anesthésique ». On évite les anesthésiques traditionnels ou on les administre à de très faibles concentrations pour prévenir l'hypotension. En outre, la réponse sympathique habituelle à l'insuffisance de l'anesthésie est souvent masquée par l'approche thérapeutique pharmacologique, ce qui rend plus difficile l'appréciation de la profondeur de l'anesthésie. Des études récentes profitent de la technique des potentiels évoqués auditifs et à d'autres méthodes pour mettre en évidence la retour à l'état de conscience avec amnésie ou de perception inconsciente. Ces techniques peuvent nous fournir des explications sur le rappel spontané plus susceptible de survenir quand on se fie à des agents non anesthésiques pour faire de l'anesthésie. De plus, certaines données montrent qu'à des concentrations inférieures au MAC, les anesthésiques n'abolissent pas de rappel spontané proportionnellement à leur indice de MAC, ce qui prouverait que tous les anesthésiques ne sont pas sur le même pied vis-à-vis la mémoire.

Il est reconnu que les morphiniques ne sont pas des anesthésiques. L'effet 'plafonne avec la fentanyl, ${ }^{9}$ le sufentanil $1^{10}$ et l'alfentanil ${ }^{11}$ en rapport avec leur capacité de réduire le MAC des agents d'inhalation ou de procurer une anesthésie adéquate. Pendant l'anesthésie au sufentanil seul pour l'induction et l'entretien (après prémédication à la morphine, scopolamine et diazépam), l'onde P3a du potentiel évoqué auditif était présente à différents stages de l'intervention, bien que l'onde N1 ait été absente, ${ }^{12}$ indiquant ainsi que le sufentanil diminue l'arousal mais que la discrimination aux changements de volume sonore était conservée, vraisemblablement comme consequence de la perception du stimulus par le patient.

Dans le but de maintenir la stabilité hémodynamique, plusieurs anesthésistes : associent des benzodiazépines aux morphiniques à la place des agents conventionnels. Cependant, bien que les benzodiazépines soient capables de produire l'amnésie, elles ne peuvent être considérées comme des agents anesthésiques. Au cours d'une étude réalisée en chirurgie cardiaque où le fentanyl était potentialisé pour l'entretien de l'anesthésie par du protoxyde d'azote et du flunitrazépam, de l'isoflurane ou du propofol, ${ }^{13}$ les potentiels auditifs évoqués de latence moyennes (MLAEP) ont subi une baisse plus importante avec le propofol ou l'isoflurane que le flunitrazépam. Au cours d'une autre étude, ${ }^{14}$ l'anesthésie a été maintenue avec de fortes doses de fentanyl avec suppléments de flunitrazépam, d'isoflurane ou de propofol. Les MLAEP ont été monitorés et on a fait tourner une cassette contenant une épreuve auditive implicite. Sept des 30 patients ont confirmé un rappel implicite. Chez tous ces patients, mais chez seulement deux des 23 autres, la latence de l'onde $\mathrm{Pa}$ a augmenté d'au moins $12 \mathrm{~ms}$ et chez tous les patients sans rappel implicite, on a constaté une forte atténuation ou une abolition de la forme d'onde des MLAEP. Le rappel implicite est survenu chez cinq patients sur dix de ceux qui avaient reçu du flunitrazépam, mais seulement chez deux sur vingt de ceux qui avaient reçu le propofol ou l'isoflurane.

Des études ont aussi été conduites pendant des interventions non cardiaques. Pendant des interventions gynécologiques avec induction au midazolam, au diazépam ou au flunitrazépam, ${ }^{15}$ on n'a pas noté de changement dans l'amplitude ou la latence de MLAEP, confirmant ainsi que cortex continuait de traiter les stimuli auditifs. Chez des patients subissant une laparotomie, ${ }^{16}$ l'anesthésie épidurale a été utilisée pour bloquer les sensations douloureuses et l'anesthésie générale maintenue avec du propofol, de l'isoflurane ou de flunitrazépam associés à du fentanyl. Les MLAEP ont été considérablement atténués ou abolis dans les deux premiers groupe mais pas dans le dernier. Des mouvements dirigés ou de la toux, considérés comme des signes d'éveil moteurs, sont survenus plus souvent dans le groupe flunitrazépam/fentanyl. En utilisant la technique de l'avant-bras isolé (un test de conscience avec amnésie), Russell ${ }^{17,18}$ a noté que $72 \%$ des patients répondaient aux ordres pendant la chirurgie lorsqu'ils étaient sous midazolam et alfentanil, $44 \%$ sous protoxyde d'azote et alfentanil, mais seulement $7 \%$ sous perfusion continue d'étomidate et de fentanyl.

Si nous acceptons que les quatre stages définis par Jones et Konieczko ${ }^{1}$ représentent un continuum, il faut inévitablement arriver à la conclusion que les mélanges de médicaments qui provoquent une incidence élevée d'état de conscience ou de rappel implicite, ou des forme d'ondes de MLAEP trouvées chez des patients qui ont une rappel implicite, sont associés à un risque plus grand de rappel spontané. Seuls les agents anesthésiques volatils ou intraveineux abolissent ces réponses.

Pour compliquer la situation, la quantité d'anesthésique requise pour prévenir le rappel spontané varie avec les associations d'analgésiques et le pourcentage du MAC peut différer selon le médicament utilisé (ou pour les anesthésiques intraveineux, de l' $\mathrm{EC}_{50}$ sur l'abolition des mouvements). Chez des volontaires non stimulés, Dwyer et al. ${ }^{19}$ ont montré que $1{ }^{\prime} \mathrm{EC}_{50}$ nécessaire pour abolir la mémoire était de 0,20 MAC pour l'isoflurane, de 0,50 MAC pour le protoxyde d'azote; des concentrations téléexpiratoires de $0,38 \mathrm{MAC}$ et de 0,64 MAC respectivement préviennent les mouvements volontaires commandés chez $50 \%$ des sujets. En ce qui concerne l'abolition de la réponse hémodynamique, le protoxyde d'azote à $70 \%$ réduit la concentration plasma- 
tique de l'alfentanil d'environ $80 \% .^{20,21}$ Cependant, pour la prévention du mouvement de réponse à la stimulation chirurgicale, le protoxyde d'azote à $67 \%$ réduit la concentration de propofol par seulement $33 \%,{ }^{22}$ mais réduit le MAC des agents volatils proportionnellement à la somme du MAC des produits administrés. Il est donc préférable de considérer le protoxyde d'azote en premier lieu comme un analgésique plutôt qu'un anesthésique.

La rappel spontanée est-il important? Le réponse est catégorique: oui. On évalue la proportion des patients qui souffrent de douleur ou de malaise pendant une période de conscience entre $10 \%{ }^{23}$ et $82 \% .^{24}$ Quiconque doute de l'intensité de la douleur ressentie pendant une intervention réalisée avec une anethésie insuffisante devrait lire les horreurs publiées. ${ }^{24,25}$ L'audition est la sensation dont on se souvient le plus fréquemment; elle ne cause pas toujours de la détresse mais peut être très désagréable si le personnel de la salle d'opération tient sur le patient des propos dérisoires, ou si leur conversation est frivole. Plusieurs patients sont conscients d'être paralysés, ce qui provoque de l'anxiété, de la panique, de l'impuissance et du découragement. ${ }^{26}$ La nature inattendue de ces expériences pénibles peut provoquer une névrose traumatique identique à celle de stress posttraumatique. Ces patients sont sujets à des attaque d'anxiété, des retours en arrière, des cauchemars, des désordres du sommeil et peuvent acquérir une crainte morbide des médecins et des hôpitaux. Une étude rapporte que $70 \%$ des patients qui avaient eu des rappels spontanés ont développé des désordres psychologiques. Il faut noter que dans la description originale par Blacher de la névrose traumatique associée avec l'éveil sous anethésie, ${ }^{27}$ aucun des patients n'avait décrit de rappel spontané; ceci confirme ma conviction qu'on ne peut se fier à des agents qui produisent de l'amnésie comme les benzodiazépines pour produire une anesthésie efficace. Seulement un tiers des patients racontent l'épisode de rappel spontané à leur anesthésiste. Même lorsque l'épisode est rapporté, le personnel médical est rarement enclin à la sympathie et gère mal la situation..$^{28}$

La grande majorité des incidents de conscience peranesthésique sont le résultat de techniques fautives. ${ }^{29}$ Cependant, présentement, la concentration exacte des agents anesthésiques requise pour garantir l'abolition du rappel spontané est inconnue. Le protoxyde d'azote à $70 \%$ (environ MAC 1 à $0,65 \%$ ) est insuffisant. ${ }^{30}$ L'isoflurane MAC 1 prévient la connaissance implicite pendant la chirurgie ${ }^{31}$ et dépasse vraisemblablement la concentration requise pour abolir le rappel spontané. Cependant, avec des techniques équilibrées qui combinent des analgésiques, de l'anesthésie régionale, des benzodiazépine à des agents anesthésiques, les permuta- tions possibles sont nombreuses et le risque de rappel spontané avec chacune de celles-ci ne peut être évalué qu'avec des études prospectives mobilisant un grand nombre de patients. Il apparaît plausible qu'une association de produits qui abolit les mouvements pendant la chirurgie ait le même effet préventif sur le rappel spontané associé à de la détresse. Quand des techniques dont on ignore le risque de reprise de conscience sont utilisées, il semble prudent d'utiliser des curarisants pour produire une relaxation plutôt qu'une paralysie et de monitorer le mouvement sous toutes ses formes. Si cela est impossible, il faut utiliser une technique reconnue pour éviter le rappel spontané des événements peropératoires.

\section{References}

1 Jones JG, Konieczko $K$. Hearing and memory in anaesthetised patients (Editorial). BMJ 1986; 292: 1291-3.

2 Sebel PS, Bonke B, Winograd E (Eds.) Memory and Awareness in Anesthesia. Englewood Cliffs, NJ, PTR Prentice-Hall, 1993.

3 Liu WHD, Thorp TAS, Graham SG, Aitkenhead AR. Incidence of awareness with recall during general anaesthesia. Anaesthesia 1991; 46: 435-7.

4 Lyons $G$, Macdonald R. Awareness during Caesarean section. Anaesthesia 1991; 46: 62-4.

5 Phillips AA, McLean RF, Devitt JH, Harrington EM. Recall of intraoperative events after general anaesthesia and cardiopulmonary bypass. Can J Anaesth 1993; 40: 922-6.

6 Goldmann $L$, Shah $M V$, Hebden $M W$. Memory of cardiac anaesthesia. Psychological sequelae in cardiac patients of intra-operative suggestion and operating room conversation. Anaesthesia 1987; 42: 596-603.

7 Mora CT, Dudeck C, Torjman MC, White PF. The effects of anesthetic technique on the hemodynamic response and recovery profile in coronary revascularization patients. Anesth Analg 1995; 81: 900-10.

8 Gilron I, Solomon P, Plourde G. Unintentional intraoperative awareness during sufentanil anaesthesia for cardiac surgery. Can J Anaesth 1996; 43: 295-8.

9 Schweiger IM, Hall RI, Hug CC Jr. Less than additive antinociceptive interaction between midazolam and fentanyl in enflurane-anesthetized dogs. Anesthesiology 1991; 74: 1060-6.

10 Hall RI, Murphy MR, Hug CC Jr. The enflurane sparing effect of sufentanil in dogs. Anesthesiology 1987; 67: 518-25.

11 Hug CC Jr, Hall RI, Angert KC, Reeder DA, Moldenhauer $C C$. Alfentanil plasma concentration vs effect relationships in cardiac surgical patients. Br J Anaesth 1988; 61: 435-40.

12 Plourde G, Joffe D, Villemure C, Trahan $M$. The P3a wave of the auditory event-related potential reveals regis- 
tration of pitch change during sufentanil anesthesia for cardiac surgery. Anesthesiology 1993; 78: 498-509.

13 Haessler R, Schwender D, Leppmeier U, Klasing S, Rindfleisch F, Peter $K$. Anaesthesia for coronary artery bypass grafting: opioid-analgesia combined with either flunitrazepam, propofol or isoflurane. Acta Anaesthesiol Scand 1993; 37: 532-40.

14 Schwender D, Kaiser A, Klasing S, Peter K, Poppel E. Midlatency auditory evoked potentials and explicit memory in patients undergoing cardiac surgery. Anesthesiology 1994; 80: 493-501.

15 Schwender D, Klasing S, Madler C, Poppel E, Peter K. Effects of benzodiazepines on midlatency auditory evoked potentials. Can J Anaesth 1993; 40: 1148-54.

16 Schwender D, Faber-Zullig E, Klasing S, Poppel E, Peter $K$. Motor signs of wakefulness during general anaesthesia with propofol, isoflurane and flunitrazepam/fentanyl and midlatency auditory evoked potentials. Anaesthesia 1994; 49: 476-84.

17 Russell IF. Comparison of wakefulness with two anaesthetic regimens. Total iv vs balanced anaesthesia. $\mathrm{Br} \mathrm{J}$ Anaesth 1986; 58: 965-8.

18 Russell IF. Midazolam-alfentanil: an anaesthetic? An investigation using the isolated forearm technique. $\mathrm{Br} J$ Anaesth 1993; 70: 42-6.

19 Dwyer R, Bennett HL, Eger EI II, Heilbron D. Effects of isoflurane and nitrous oxide in subanesthetic concentrations on memory and responsiveness in volunteers. Anesthesiology 1992; 77: 888-98.

20 de Lange S, de Bruijn NP. Alfentanil-oxygen anaesthesia: plasma concentrations and clinical effects during variablerate continuous infusion for coronary artery surgery. $\mathrm{Br} \mathrm{J}$ Anaesth 1983; 55: 183S-189S.

21 Ausems ME, Hug CC Jr, Stanski JR, Burm AG. Plasma concentrations of alfentanil required to supplement nitrous oxide anesthesia for general surgery. Anesthesiology 1986; 65: 362-73.

22 Davidson JA, Macleod AD, Howie JC, White M, Kenny GN. Effective concentration 50 for propofol with and without $67 \%$ nitrous oxide. Acta Anaesthesiol Scand 1993; 37: 458-64.

23 Crawford JS. Principles and Practice of Obstetric Anaesthesia. Blackwell Scientific Publications, Oxford, 1984; 297.

24 Evans JM. Patients' experiences of awareness during general anaesthesia, In: Rosen M, Lunn JN (Eds.). Consciousness Awareness and Pain in General Anaesthesia. London: Butterworths, 1987: 184-92.

25 Anonymous. On being aware (Editorial). $\mathrm{Br} \mathrm{J}$ Anaesth 1979; 51: 711-2.

26 Moerman N, Bonke B, Oosting J. Awareness and recall during general anesthesia. Facts and feelings. Anesthesiology 1993; 79: 454-64.
27 Blacher RS. On awakening paralysed during surgery. A syndrome of traumatic neurosis. JAMA 1975; 234: 67-8.

28 Cobcroft MD, Forsdick C. Awareness under anaesthesia: the patients' point of view. Anaesth Intensive Care 1993; 21: 837-43.

29 Hargrove RL. Awareness under anaesthesia. Journal of the Medical Defence Union 1987; 9-11.

30 Harris TJ, Brice DD, Hetherington RR, Utting JE. Dreaming associated with anaesthesia: the influence of morphine premedication and two volatile adjuvants. $\mathrm{Br} \mathrm{J}$ Anaesth 1971; 43: 172-8.

31 Dwyer R, Bennett HL, Eger EI II, Peterson N. Isoflurane anesthesia prevents unconscious learning. Anesth Analg 1992; 75: 107-12. 